\title{
REPRESENTAÇÕES SOCIAIS DE PROFISSIONAIS DE UNIDADES DE PRONTO ATENDIMENTO SOBRE O SERVIÇO MÓVEL DE URGÊNCIA ${ }^{1}$
}

\author{
Meiriele Tavares Araújo², Marília Alves³, Maria Flávia Carvalho Gazzinellit, Thais Batista da Rocha
}

${ }^{1}$ Artigo extraído da dissertação - Representações Sociais dos Profissionais de Saúde das Unidades de Pronto Atendimento sobre
o Serviço de Atendimento Móvel de Urgência, apresentada ao Programa de Pós-Graduação em Enfermagem da Universidade
Federal de Minas Gerais (UFMG), 2010.
${ }^{2}$ Doutoranda em Enfermagem pela Escola de Enfermagem da UFMG. Enfermeira da Prefeitura Municipal de Betim-MG.
Docente do Curso de Enfermagem da Faculdade de Pará de Minas. Minas Gerais, Brasil. E-mail: enfaraujo@gmail.com
${ }^{3}$ Doutora em Enfermagem. Professora Titular do Departamento de Enfermagem Aplicada da Escola de Enfermagem da UFMG.
Pesquisador CNPq. Minas Gerais, Brasil. E-mail: marilix@enf.ufmg.br
${ }^{4}$ Doutora em Enfermagem. Professor Adjunto do Departamento de Enfermagem da UFMG. Minas Gerais, Brasil. E-mail:
flavia@enf.ufmg.br
${ }^{5}$ Mestranda em Enfermagem pela Escola de Enfermagem da UFMG. Minas Gerais, Brasil. E-mail: thayslalinha@hotmail.com

RESUMO: Este artigo objetivou analisar as representações sociais dos profissionais de saúde das Unidades de Pronto Atendimento sobre Serviço de Atendimento Móvel de Urgência, utilizando Teoria das Representações Sociais e Teoria do Núcleo Central. Foram sujeitos 274 profissionais, de quatro Unidades de Pronto Atendimento de Belo Horizonte-MG, sendo os dados coletados por meio de questionário. No núcleo central, os termos emergência, etilista, rapidez, resgate, transporte e urgência refletem uma visão positiva e funcional do Serviço de Atendimento Móvel de Urgência. Na primeira periferia, as palavras "casos graves" e "trauma" reforçam esse núcleo. Na terceira periferia, agilidade, atendimento, conflito, equipe despreparada, pré-hospitalar e salvar vidas revelam tensões e conflitos existentes. Na segunda periferia, habilidade, humanização, precária e regulação mostram o serviço como hábil, humanizador e, ao mesmo tempo, precário e falho na regulação. Conclui-se que as representações sociais desse serviço, embora em maior parte, positivas, possuem aspectos que afetam as práticas e o funcionamento desse serviço.

DESCRITORES: Serviços médicos de emergência. Pessoal de saúde. Atenção secundária a saúde. Transporte de pacientes. Ambulâncias.

\section{SOCIAL REPRESENTATIONS OF EMERGENCY CARE UNIT PROFESSIONALS ON EMERGENCY MOBILE SERVICES}

\begin{abstract}
This article aims to analyze social representations about Emergency Mobile Services from health professionals who work in Emergency Care Units using the Social Representations Theory and the Central Nucleus Theory. The subjects were 274 health professionals from four Emergency Care Units in Belo Horizonte, MG, Brazil. The data was collected through questionnaire application. At the core, the terms emergency, alcoholic, speed, rescue, transportation, and emergency reflect a positive and functional perception of emergency mobile service care. In the first periphery, the words "severe cases" and "trauma" reinforce these core elements. In the third periphery, agility, customer service, conflict, unprepared-team, pre-hospital, and saving lives reveal existing tensions and conflicts. In the second periphery, ability, humanization, precarious, and regulation show the service as skillful, humanizing, and at the same time precarious and flawed in its regulation. We conclude that social representations of this service, while positive in their majority, possess aspects which affect the practices and functioning of this service.
\end{abstract}

DESCRIPTORS: Emergency medical services. Health professional. Secondary health care. Transportation of patients. Ambulances

\section{REPRESENTACIONES SOCIALES DE LOS PROFESIONALES DE LAS UNIDADES DE EMERGENCIA ACERCA DEL SERVICIO MÓVIL DE URGENCIA}

RESUMEN: En este artículo se pretende analizar las representaciones sociales de los profesionales de salud de Unidades de Emergencia acerca del Servicio de Atención Móvil de Urgencia con base en la Teoría de las Representaciones Sociales y la Teoría del Núcleo Central. Los sujetos del estudio son 274 profesionales de salud de cuatro Unidades de Emergencia de Belo Horizonte-MG. La recolección de los datos se hizo por medio de una encuesta. En el núcleo central del cuadro las palabras: emergencia, alcohol, rapidez, rescate, transporte y urgencia reflejan una visión positiva y funcional del Servicio de Atención Móvil de Urgencia. En la primera periferia se encuentran las palabras casos graves y trauma que refuerzan los elementos del núcleo. En la tercera periferia, agilidad, atención, conflicto, equipo sin preparación, prehospitalario y salvar vidas revelan las tensiones y conflictos existentes. En la segunda periferia: habilidad, humanización, precariedad y falla en la regulación muestran el Servicio Móvil de Urgencia como ágil y humano, y al mismo tiempo, deficiente y con fallas en la Regulación. Se concluye que las representaciones sociales del Servicio Móvil de Urgencia, aunque en su mayoría son positivas, presentan obstáculos para sus prácticas y funcionamiento de ese servicio.

DESCRIPTORES: Servicios médicos de urgencia. Personal de salud. Atención secundaria a la salud. Transporte de pacientes. Ambulancias. 


\section{INTRODUÇÃO}

A sociedade moderna caracteriza-se por estilos de vida diferentes dos observados no passado e que, frequentemente, dão origem a problemas de saúde. Entre esses problemas, encontra-se a alta mortalidade por causas externas, que se tornam cada vez mais alarmantes. A mudança no perfil de morbimortalidade fez com que as causas externas, destacando-se os acidentes e violências, passassem a ser considerados problemas de saúde pública, demandando ações governamentais para sua redução. ${ }^{1}$ Essa mudança fez com que a área de urgência e emergência se destacasse no Sistema Único de Saúde (SUS).

De acordo com a Classificação Internacional de Doenças (CID), causas externas são um conjunto de eventos como acidentes devidos ao trânsito, trabalho, quedas, envenenamentos, afogamentos, ferimentos, fraturas, queimaduras, intoxicações, violências e as causas intencionais (agressões e lesões autoprovocadas), podendo ou não levar a óbito. ${ }^{2}$ No Brasil, atualmente, as causas externas ocupam o quarto lugar entre todas as causas de morte. Dos 1.031.691 óbitos ocorridos em 2006, $13,6 \%$ foram por causas externas, sendo que o coeficiente de mortalidade nesse ano também revelou que $19,9 \%$ desses óbitos foram devidos a acidentes de trânsito e 26,3 \% a agressões. ${ }^{3}$

$\mathrm{O}$ aumento dos acidentes e da violência urbana, associado à insuficiente estruturação da rede de serviços de saúde, para atender as urgências e emergências, contribui para aumentar a sobrecarga de trabalho e a precariedade das Unidades de Saúde que fazem esse tipo de atendimento. No entanto, as demandas de atenção às urgências não são somente decorrentes de causas externas, mas também de outros agravos relacionados à mudança do padrão demográfico, ao envelhecimento da população e ao aumento das doenças crônicas não transmissíveis ou de quadros agudos que exigem abordagens diferenciadas. ${ }^{4}$

As demandas por atendimentos de urgência e emergência pressionam os Serviços de saúde no sentido de se organizarem para atender pacientes graves que necessitam de maior aporte tecnológico e maior habilidade profissional. Em resposta a essas necessidades, o Ministério da Saúde (MS) instituiu o Regulamento Técnico dos Sistemas Estaduais de Urgência e Emergência com a Portaria n ${ }^{\circ} 2.048$ GM/MS de 2002 e a Política Nacional de Atenção às Urgências com a Portaria n 1.863 GM/MS de 2003. Essas Portarias determinam a organização de redes loco regionais de atenção integral às urgências, como peças interligadas da trama de manutenção da vida, organizadas nos componentes: pré-hospitalar fixo, pré-hospitalar móvel, hospitalar e pós-hospitalar ${ }^{1}$.

O Atendimento Pré-Hospitalar (APH) fixo é composto pelas Unidades Básicas de Saúde (UBS), Unidades de Saúde da Família (USF), ambulatórios especializados, serviços de diagnóstico e terapias e unidades não hospitalares de atendimento às urgências. Essas unidades são responsáveis pelo atendimento a casos de urgência por demanda espontânea, referenciados pelas UBS e Unidades de atendimento pré-hospitalar móvel. A Portaria $\mathrm{n}^{\circ}$ 1.864/GM regulamentou o componente Pré-Hospitalar Móvel, definindo sua estruturação, por meio da implantação do Serviço de Atendimento Móvel de Urgência (SAMU) e dos serviços associados de salvamento e resgate, em todo o território nacional com as Centrais de Regulação Médica acessadas pelo número 192 e os Núcleos de Educação em Urgência. ${ }^{1}$

OSAMU possui responsabilidades e diretrizes que contemplam medidas de promoção da saúde e prevenção de doenças mediante a articulação de diferentes segmentos sociais. Nesse contexto, o atendimento a urgências e emergências foi implantado em todo o território nacional mediante parcerias do MS com as Secretarias Estaduais e Municipais de Saúde. A atenção às urgências é realizada por meio da articulação dos diversos níveis de atenção, estando presente desde a atenção básica até a hospitalar. ${ }^{5}$ Essa articulação torna-se importante pois, quando ocorre um dano a saúde, o socorro adequado contribui para minimizar as sequelas e melhorar o prognóstico da vítima.

Assim, o SAMU é considerado um componente organizador da assistência, capaz de responder a demandas de urgências no domicílio, no local de trabalho, em vias públicas e onde o usuário doSUS precisar, com recursos necessários e adequados para a complexidade de sua condição. ${ }^{5} \mathrm{O}$ Serviço é responsável por atendimentos a usuários com demandas clínicas, obstétricas e psiquiátricas, tendo como retaguarda outros Serviços de urgência e emergência como as Unidades de Pronto Atendimento (UPA) e os Hospitais de alta complexidade. Entretanto, essas Unidades de retaguarda geralmente trabalham com sua capacidade de produção acima do previsto no planejamento anterior à criação do SAMU. ${ }^{6}$

Mas os profissionais das UBS ainda não reconhecem sua responsabilidade no atendimento às urgências. Ressaltam como fatores dificultadores, a 
inadequação da estrutura física, a falta de materiais e de tecnologias, além do despreparo da equipe. $\mathrm{O}$ SAMU, por outro lado, possui critérios específicos de atendimento às urgências, pessoal especializado e grande autonomia, o que faz com que, em algumas situações, se recuse a atender à solicitação da UBS, motivo de conflitos entre os dois Serviços e de dificuldade para a realização da assistência integral. ${ }^{7}$

Em relação às UPA, observa-se que os profissionais trabalham com grande demanda espontânea e uma percepção de prestação de assistência negativa da população, principalmente pela demora no atendimento. ${ }^{6}$ Além disso, os profissionais das UPA apresentam dificuldades para estabelecer parcerias e reconhecer a legitimidade do SAMU no julgamento das situações de urgência que devem atender e que são semelhantes às observadas na UBS. Assim, a forma como os profissionais das UPA representam o SAMU relacionam-se intimamente a seu contexto de trabalho especifico. Em determinadas situações, percebe-se que representam o trabalho do SAMU como bom e essencial para o funcionamento da rede de Serviços de saúde; em outras, como aquele que traz sobrecarga de trabalho, comprometendo a organização do processo de atendimento. São as chamadas representações sociais ligadas a sistemas de pensamento social, que perduram no tempo e possuem estabilidade

A representação social é um sistema de valores, de noções e de práticas que possuem uma dupla tendência. Uma tendência seria a de instaurar uma ordem que dá aos indivíduos a possibilidade de se orientarem no ambiente social e material, de dominá-lo. A outra tendência seria a de assegurar a comunicação entre os membros de uma comunidade propondo-lhes um código para suas trocas. ${ }^{8}$

As representações sociais dos profissionais das UPA sobre o SAMU podem ter algumas particularidades, se comparadas às de profissionais de outros Serviços, em decorrência das condições locais de trabalho e de como se dão as relações. Entre essas condições, encontram-se o atendimento de grande demanda espontânea de pacientes em situação de urgência ou não, a oscilação no quadro de profissionais e a falta de leitos de retaguarda em hospitais para receber os pacientes graves que permanecem em observação na Unidade por tempo maior que o previsto. Além disso, as UPA são obrigadas a receber usuários encaminhados pelo SAMU, o que lhes acrescenta novos casos graves e problemas ao cotidiano de trabalho. Assim, este estudo teve como finalidade responder à seguinte questão: quais são as representações sociais dos profissionais das UPA sobre o SAMU?

O estudo teve por objetivo analisar as representações sociais dos profissionais de saúde das Unidades de Pronto Atendimento sobre o SAMU, por meio da abordagem estrutural das representações.

\section{PERCURSO METODOLÓGICO}

Este estudo fundamentou-se na Teoria das Representações Sociais ${ }^{9}$ e na Teoria do Núcleo Central $^{10}$, na perspectiva da Psicologia Social, utilizando-se do software Ensemble de programmes permettant l'analyse des evocations - versão 2003 (EVOC 2003) como método para a análise dos dados.

Foi desenvolvido em quatro UPA da SMSA de Belo Horizonte, sendo selecionados de acordo com a realização de atendimento dos usuários provenientes do SAMU. Os sujeitos da pesquisa foram 274 profissionais de saúde das UPA que recebem usuários encaminhados pelo SAMU, sendo 89 médicos, 31 enfermeiros e 154 profissionais de enfermagem (auxiliares e técnicos). O número de sujeitos foi considerado suficiente quando os dados foram coletados nos três plantões, durante o dia e à noite, considerando a carga horária de trabalho de 12 por 60 horas de descanso da equipe de enfermagem. A opção por acompanhar a escala dos profissionais de enfermagem deveu-se ao fato de que somente esses profissionais tinham uma lógica de intervalos de trabalho que possibilitava a coleta de dados sistematizados. Os sujeitos foram escolhidos aleatoriamente, de acordo com o plantão.

O projeto de pesquisa foi avaliado e aprovado pela Câmara do Departamento de Enfermagem Aplicada da Escola de Enfermagem (ENA/EE) da Universidade Federal de Minas Gerais (UFMG) e pelos Comitês de Ética em Pesquisa da UFMG (Parecer $n^{\circ}$ 215/08) e da Secretaria Municipal de Saúde de Belo Horizonte (Parecer no 14/2008), de acordo com a Resolução 196/96 do Conselho Nacional de Saúde. ${ }^{11}$

A coleta de dados foi realizada por meio de questionário, com itens que identificavam o perfil dos entrevistados e as evocações livres sobre o termo indutor SAMU. Foram coletados 274 questionários, totalizando 1338 palavras diferentes evocadas que foram agrupadas em 85 palavras padronizadas. Os dados correspondentes ao perfil dos sujeitos foram organizados num banco de dados no Microsoft Excel que permitiu a análise por meio de frequências simples das variáveis. 
A organização dos dados referentes à Representação Social foi realizada por meio do software EVOC 2003. Este programa calcula e informa a frequência simples de ocorrência de cada palavra evocada, a média ponderada de ocorrência de cada palavra e a média das ordens médias ponderadas do conjunto dos termos evocados, o que possibilita estratificar essas evocações em núcleo central e periférico.

O quadro de quatro casas criado discrimina o núcleo central (elementos mais frequentes e mais importantes situados no quadrante superior esquerdo), os elementos intermediários ou $1^{\text {a }}$ periferia (elementos periféricos mais importantes situados no quadrante superior direito), os elementos de contraste (com baixa frequência, mas considerados importantes pelos sujeitos, situados no quadrante inferior esquerdo) e os elementos periféricos da representação ou $2^{\mathrm{a}}$ periferia (menos frequentes e menos importantes, localizados no quadrante inferior direito). ${ }^{12}$ Para a interpretação dos dados, realizou-se a leitura das características representacionais do conjunto da população para o termo indutor SAMU.

\section{APRESENTAÇÃO E DISCUSSÃO DOS RESULTADOS}

Os dados de caracterização do perfil dos sujeitos da pesquisa mostram que $67 \%$ dos profissionais são do feminino, 31,1\% do sexo masculino, e 1,9\% não declararam. A maioria dos profissionais possui mais de 25 anos (93\%), casada $(45,6 \%)$ seguidos de $37,6 \%$ de solteiros, $11,3 \%$ de divorciados, $1,8 \%$ viúvos e $3,7 \%$ não especificaram situação civil. Em relação às categorias profissionais, 56,2\% eram auxiliares e técnicos de enfermagem, $32,5 \%$ médicos e $11,3 \%$ enfermeiros.

Considerando o tempo de formado, $74,4 \%$ mais de cinco anos de formados, $18,7 \%$ tinham entre um e quatro anos, 5,5\% tinham menos de um ano e 1,5\% não declararam. Entre os profissionais de nível superior, $62,5 \%$ possuem pós-graduação, $25 \%$ não possuem pós-gradução e $10 \%$ estão cursando a pós-graduação. Em relação ao tempo de trabalho na UPA 39,2\% tinham mais cinco anos, 23,4\% estavam entre um e quatro anos, $36,3 \%$ tinham menos de um ano e 1,1\% não souberam responder, existindo equilíbrio entre os considerados novatos no serviço e aqueles já com maior experiência.

O corpus para a análise das Representações Sociais foi formado por 1338 palavras. Dessas 1338 apenas 108 foram diferentes e foram agrupadas em 85 palavras padronizadas. A média das Ordens Médias de Evocação (OME) foi igual a 2.95, sendo arredondada para três. A frequência média foi 44 e a mínima 23. Dessas palavras evocadas, 28 foram ditas apenas uma vez e a palavra "casos-graves", de maior freqüência de evocação, foi dita 74 vezes. No quadro de quatro casas, tabela 1 , construído a partir do EVOC 2003 com as evocações ao termo indutor SAMU, pode-se observar o conteúdo das representações sociais, bem como a estrutura e a hierarquia dos elementos de seus sistemas cognitivos.

Tabela 1 - Quadro de quatro casas relativo à estrutura representacional de profissionais de saúde acerca do SAMU. Belo Horizonte-MG, 2009

\begin{tabular}{|c|c|c|c|c|c|c|}
\hline \multicolumn{4}{|c|}{$<3$} & \multicolumn{3}{|c|}{$>=3$} \\
\hline Freq.média & Termo evocado & Freq & OME & Termo evocado & Freq & OME \\
\hline \multirow{7}{*}{$>=44$} & \multicolumn{3}{|c|}{$\begin{array}{c}\text { Quadrante superior esquerdo } \\
\text { Núcleo central } \\
\end{array}$} & \multicolumn{3}{|c|}{$\begin{array}{c}\text { Quadrante superior direito } \\
1^{\mathrm{a}} \text { periferia } \\
\end{array}$} \\
\hline & Emergência & 48 & 2,104 & Casos-graves & 74 & 3,108 \\
\hline & Etilista & 65 & 2,200 & Trauma & 49 & 3,245 \\
\hline & Rapidez & 57 & 2,842 & & & \\
\hline & Resgate & 46 & 2,261 & & & \\
\hline & Transporte & 69 & 2,826 & & & \\
\hline & Urgência & 62 & 2,145 & & & \\
\hline \multirow{7}{*}{$<44$} & \multicolumn{3}{|c|}{$\begin{array}{c}\text { Quadrante inferior esquerdo } \\
\text { Elementos constrastante ou intermediários }\end{array}$} & \multicolumn{3}{|c|}{$\begin{array}{l}\text { Quadrante inferior direito } \\
\text { 2 }^{\text {a }} \text { Periferia }\end{array}$} \\
\hline & Agilidade & 31 & 2,677 & Habilidade & 35 & 3,486 \\
\hline & Atendimento & 36 & 2,361 & Humanização & 43 & 3,233 \\
\hline & Conflito & 23 & 2,957 & Precário & 26 & 3,269 \\
\hline & Equipe-despreparada & 41 & 2,878 & Regulação & 27 & 3,333 \\
\hline & Pré-hospitalar & 30 & 2,833 & & & \\
\hline & Salvar-vidas & 28 & 2,964 & & & \\
\hline
\end{tabular}


No quadrante superior esquerdo, encontram-se os possíveis elementos do núcleo central da representação deste estudo, as palavras que possuem maiores frequências e menores OME, e que correspondem àqueles elementos mais importantes. As palavras do núcleo central refletem uma imagem do SAMU em sua maior parte positiva e de reconhecimento de seu trabalho. Esses elementos destacam o caráter funcional do SAMU, referindo-se às características descritivas e à inscrição do objeto nas práticas sociais dos sujeitos deste estudo. OSAMU é entendido como um tipo de assistência pré-hospitalar móvel caracterizado pelo atendimento rápido, ou seja de resgate ou transporte, de pacientes com quadros de urgência ou emergência declarados. O elemento etilista aparece como fator inesperado e destoante.

O elemento com maior destaque entre os centrais é transporte, esta ideia está ligada ao atendimento pré-hospitalar que se iniciou com a concepção inicial de transporte dos pacientes para um local onde pudessem receber o atendimento necessário para garantir-lhes a vida. ${ }^{13}$ No entanto, essa ideia deve ir além do transporte da vítima, de acordo com seu status, de forma segura, para a Unidade de referência adequada deve incluir, durante o percurso, a garantia dos cuidados necessários, preconizados pelo protocolo de atendimento pré-hospitalar a fim de garantir-lhes a vida ou minimizar-lhes os danos.

Dentro da padronização do termo transporte, também foram evocados os termos carona, entrega, ótimo transportador, taxi e ônibus que, dentre outros, representam uma forma de questionamento dos profissionais da UPA acerca da qualidade e do tipo de atendimento prestado pelo SAMU. Assim, uma mesma ideia de transporte abriga uma perspectiva positiva e negativa do serviço. Há discordâncias com relação aos julgamentos clínicos feitos pelo SAMU por parte dos profissionais da UPA, bem como a critica aos casos que o SAMU encaminha como sendo casos-graves. ${ }^{14}$ Os profissionais de saúde da UPA ainda relatam que o SAMU é mal aproveitado por parte da população que o utiliza como "taxi", demandando um atendimento que não seria necessário. ${ }^{14}$

O segundo elemento em destaque no núcleo central foi etilista, de acordo com o julgamento desses profissionais, os pacientes alcoolizados não se caracterizam como urgência ou emergência. Em relação a esse fato, os profissionais do SAMU argumentam que os pacientes etilistas sempre constituíram uma demanda reprimida que ganhou visibilidade social e apontam que eles podem vir a se caracterizar como um quadro de urgência. ${ }^{14}$

Segundo os profissionais do SAMU, muitos cidadãos acionam a Central de Regulação ao se depararem com uma pessoa caída na rua e, quando a unidade do SAMU chega ao local, seus profissionais verificam a presença de etilismo, associado ou não a alguma outra comorbidade. ${ }^{14}$ Em algumas dessas situações, os profissionais se veem "obrigados" ou "intimidados" pela população a levar o paciente para o serviço de saúde de referência, a UPA. Dessa forma, o SAMU adquiriu a conotação de serviço descaracterizado.

O elemento urgência apresenta-se com uma frequência de 62 e OME de 2,145 seguido_da emergência com frequência de 48 e OME de 2,104. Esses elementos estão intimamente ligados ao papel desempenhado peloSAMU, bem como aos elementos seguintes da sequência do núcleo central resgate e rapidez que preveem um primeiro atendimento com um tempo limite para que a vida seja preservada ou ocorra minimização de danos ou sequelas.

A proximidade da frequência entre os elementos urgência e emergência deve-se à imprecisão, à confusão e à polêmica em torno dos conceitos de urgência e emergência. ${ }^{15}$ Define-se Urgência como situação clínica aguda, com ou sem risco potencial de vida, enquanto Emergência seria uma situação com risco iminente de vida ou que cause intenso sofrimento ao paciente. ${ }^{16}$ Mas considera-se importante destacar que tanto profissionais quanto usuários possuem percepções únicas e muitas vezes ligadas a vivencia sobre o conceito de urgência e emergência que diferem dessas concepções teóricas. ${ }^{15,22}$ A Política Nacional da Atenção ás Urgências ${ }^{1}$ reafirma essa dificuldade conceitual e técnica que existe no cotidiano dos serviços de saúde em classificar o que é urgência e emergência sugerindo que os termos sejam usados como sinônimos de forma a garantir um atendimento de qualidade em ambos os casos.

No quadrante superior direito, ou seja, primeira periferia, estão as palavras que também tiveram alta frequência, mas cuja posição média na ordem de evocação não foi suficiente para que fizessem parte do núcleo central. Nesse espaço encontram-se as palavras casos-graves e trauma que reafirmam e reforçam o papel do SAMU como um serviço de atendimento pré-hospitalar que visa realizar atendimento de urgência e emergência, como traumas e casos-graves. Na prática, existe e se concretiza a seguinte equivalência: emergência e casos traumáticos, isto é, casos que levam à suspeita da necessidade de uma intervenção rápida. ${ }^{17}$ 
Os elementos agilidade, atendimento, conflito, equipe-despreparada, pré-hospitalar e salvar-vidas encontram-se dentro do quadrante dos elementos contrastantes ou intermediários, ou seja, complementam e discutem o núcleo central e, ao mesmo tempo, representam elementos de tensão nas relações entre os serviços estudados. Esses elementos ainda podem se caracterizar como um novo sub-grupo que começa a ter um olhar mais crítico para o objeto SAMU podendo causar modificações no núcleo central identificado.

O elemento agilidade reforça e influencia o termo rapidez presente no núcleo central, caracteriza o reconhecimento, por parte dos profissionais da UPA, da eficiência do SAMU em atender as situações de urgência/emergência que demandam rapidez e habilidade.

Já o termo atendimento liga-se a todos os termos presentes no núcleo central de forma positiva, considerando-se que transporte e resgate preveem uma forma de atendimento. A palavra evocada conflito possui vários termos padronizados, que mostram as dificuldades dos profissionais das UPA em lidar com o SAMU. Tais dificuldades talvez decorram dos processos de trabalho "engessados" de ambos, considerando-se o contexto de tensão e cobrança em que desenvolvem seus papéis.

As evocações falta de educação, pepino, problemático, rabo de foguete, divergência SAMU/ bombeiro, discórdia da equipe remetem a aspectos relacionados à central de regulação, tendo em vista que o contato prévio para a comunicação de casos e a designação da Unidade que deverá receber os pacientes, ou seja, qual UPA, cabe ao médico regulador que pode utilizar sua prerrogativa de autonomia e autoridade na rede de serviços para referenciar os pacientes.

Na relação com outras áreas no contexto dos serviços de saúde, é necessário aprimorar o diálogo, buscando uma integração que rompa as barreiras estabelecidas pela dificuldade de se trabalhar em uma lógica fundamentada em relações horizontais, com pactuação e compromisso com a sociedade. ${ }^{19}$ Outros autores buscam justificar a falta de comunicação verbal pela própria característica dos serviços de urgência: foco nas ações cuja base é a condição da vítima, tempo escasso e necessidade de se evitar o risco imediato para a manutenção da vida. ${ }^{19,22}$

O elemento equipe-despreparada questiona a postura ética e a capacidade técnica dos profissionais do SAMU. Percebe-se que existe um descrédito dos profissionais da UPA em relação ao preparo e à qualificação dos profissionais do SAMU para lidar com os casos atendidos e com as relações estabelecidas com outros profissionais.

Entretanto, observa-se que os elementos "conflitos" e "equipe-despreparada" são um subgrupo dentro das representações que explicitam as dificuldades nas relações e no processo de trabalho dos dois Serviços e, ao mesmo tempo, destacam uma falta de compreensão e de aceitação dos papéis dos profissionais na rede de serviços de saúde. Apesar de ser um serviço recente, os profissionais têm maior autoridade, conferida pelo Ministério da Saúde por meio da Portarias GM/MS n ${ }^{\circ} 2048^{1}$ e GM/MS n ${ }^{\circ} 1864^{1}$, de permear todos os serviços de saúde com certa autonomia e ainda gerar maior sobrecarga de trabalho nas $\mathrm{UPA}^{7}$, ao referenciar os usuários com demandas simples.

Os elementos pré-hospitalar e salvar-vidas aparecem com alta frequência e baixa ordem de evocação média. O elemento pré-hospitalar reforça os elementos resgate e transporte, presentes no núcleo central enquanto salvar-vidas reafirma todos os elementos do núcleo central como função precípua a ser desenvolvida pelo SAMU.

As palavras localizadas no quadrante inferior direito são aquelas com menores frequências de evocação e evocadas mais tardiamente, compondo os elementos da segunda periferia da representação. Nessa periferia, encontram-se as palavras habilidade, humanização, precário e regulação. Nesse espaço da representação do SAMU, observa-se atitude positiva frente a esse serviço, reconhecido pelos profissionais da UPA como instância de humanização do atendimento. Ao mesmo tempo, atributos negativos são imputados ao SAMU visto como precário em sua estruturação e nas relações e atendimentos que realiza. Todos os elementos desse quadrante reforçam os que estão presentes na zona de contraste e direcionam questionamentos da função doSAMU até os anteriormente reconhecidos como eficientes no núcleo central. Com relação ao termo habilidade, entendido como capacidade de executar intervenções prontamente, esse é citado como uma importante competência para o atendimento em situações de urgência além do raciocínio clínico para a tomada de decisão. ${ }^{20}$

O elemento humanização influencia mais claramente o termo atendimento presente na zona de contraste e isso se deve à Política Nacional de Humanização (PNH) presente na rede de Serviços de saúde do SUS. O Serviço de urgência é caracterizado pela não formação de vínculo já que todos os usuários estão de passagem naquela 
instância para atendimentos pontuais. Destaca-se ainda que os hospitais de urgência e emergência constituem-se como um lócus privilegiado para a manifestação dos intensos processos de exclusão, violência social, e brutal banalização do sofrimento alheio, especialmente de indiferença em relação aos desfavorecidos, que marcam nossa sociedade e os profissionais..$^{21-22}$

O termo precário parece ser na realidade, uma visão projetada da UPA sobre o SAMU considerando que as USB e Unidade de Suporte Avançado de Vida (USA), Unidades de atendimento do SAMU, são reconhecidamente bem equipadas e seus profissionais treinados. Outro ponto é a dificuldade de compreensão sobre a regulação dos casos o que justifica a utilização do SAMU para alguns casos e, para outros, não.

O elemento regulação, presente na segunda periferia, leva-nos a uma discussão atual sobre o Serviço de atendimento pré-hospitalar, que seria a organização do fluxo e da demanda a serem encaminhados para os Serviços da rede. Após o acolhimento e a identificação das chamadas, as solicitações são analisadas pelo médico regulador que classifica o nível de urgência e define o recurso necessário ao seu adequado atendimento. Sua decisão pode envolver desde um simples conselho médico até o envio de uma USA ao local ou o acionamento de outros meios de apoio, se julgar necessário. ${ }^{1}$

Essa discussão traz à tona a sobrecarga dos Serviços de urgência que ainda são utilizados como porta de entrada. Nesse sentido, as Unidades de Urgência e Emergencia, enquanto um serviço de portas abertas 24 horas, tornam-se espaços privilegiados de manifestação da exclusão social e da violência. ${ }^{21-22}$

\section{CONSIDERAÇÕES FINAIS}

OSAMU constitui uma proposta viável para a melhoria dos atendimentos de primeiros socorros e transporte de pacientes para os Serviços de referência adequados. Por trazer a melhoria no atendimento e facilitar o acesso, o SAMU ganhou "fama" e reconhecimento o seio da população usuária.

Em contrapartida, essa mesma "melhoria no acesso da população" vem servindo de elemento conflitante para os profissionais das UPA que trabalham com sobrecarga de atendimentos da demanda espontânea desvinculada das UBS, equipes desfalcadas, processo de trabalho desarticulado, sucateamento da estrutura física, poucos recursos diagnósticos e dificuldades de referência e contra-referência. Nesse contexto, recebem os pacientes trazidos pelas Unidades do SAMU, os quais muitas vezes não são avaliados com os mesmos critérios adotados pelos profissionais das UPA, o que gera conflitos e discussões entre as equipes dificultando o reconhecimento do trabalho do SAMU como importante e funcional.

O SAMU é, então, representado por profissionais da UPA como um Serviço de transporte de casos de urgência e emergência que preveem ações rápidas e de resgate. $\mathrm{O}$ etilista é trazido como um questionamento à demanda que esse Serviço vem atendendo. Os elementos casos-graves e traumas reforçam o que esses profissionais de saúde entendem como urgência e emergência. Os termos agilidade, atendimento, pré-hospitalar e salvar-vidas influenciam todo o núcleo central de forma positiva e reforça as funções do SAMU. Os termos equipe-despreparada e conflito surgem como um subgrupo. Esse subgrupo traz uma visão mais crítica e questionadora do SAMU do que aquela trazida pelos demais elementos, podendo ser um ponto inicial de mudança do núcleo central estabelecido. O elemento humanização vem discutir o tipo de atendimento prestado, enquanto o termo precário reforça a visão critica colocada por meio dos elementos equipe-despreparada e conflito. E, por fim, o elemento regulação reforça o elemento atendimento e vem a ser um ponto-chave para a atuação das criticas apresentadas nos outros elementos.

Cabe destacar que os profissionais de enfermagem tanto no cotidiano de atendimento das urgências quanto sujeitos desse estudo representam a maior parte dos profissionais de saúde e pode-se inferir que boa parte dessa representação social explicitada condiz com a representação das equipes de enfermagem da UPA. Ressalta-se também que a prática de enfermagem nos serviços de urgências ainda é pouco abordada pela literatura e o mesmo também acontece nesses lócus de trabalho devendo ser analisado de forma mais aprofundada principalmente quando se discute precarização e sobrecarga de trabalho de enfermagem nesses serviços bem como na qualidade e responsabilidade ética da assistência prestada.

Acredita-se que o SAMU, enquanto uma nova forma de atendimento e serviço de urgência deve ser aprimorado e, para isso, faz-se importante considerarmos a discussão do funcionamento das redes de referência e contra-referência e sua organização, de forma a melhorar as relações entre os profissionais dos dois Serviços, bem como as formas de regulação do SAMU. Assim, o SAMU é 
reconhecido como um serviço de excelente competência técnica e de grande alcance, mas que ainda necessita de aperfeiçoamento de sua capacidade gerencial e organizacional, elementos que poderão ser objetos de novas pesquisas.

\section{REFERÊNCIAS}

1. Ministério da Saúde (BR). Política Nacional de Atenção às Urgências. $3^{\mathrm{a}}$ ed.ampl. Brasília (DF): MS; 2006.

2. Organização Mundial da Saúde. CID-10 Classificação estatística internacional de doenças e problemas relacionados à saúde. São Paulo (SP): USP; 1997.

3. Ministério da Saúde (BR). Banco de dados do Sistema Único de Saúde-DATASUS [online]. Brasília (DF): MS; 2008 [acesso 2009 Ago 10]. Disponível em http://www.datasus.gov.br/idb

4. Carvalho BKL de. A rede de urgência em Belo Horizonte-MG-Brasil. Rev Médica Minas Gerais. 2008 Out-Dez;18(4):275-8.

5. Vieira CMS, Mussi FC. A implantação do projeto de atendimento Móvel de Urgência em Salvador/ BA: panorama e desafios. Rev Esc Enferm USP. 2008 Dez; 42(4):793-7.

6. Rocha AFS. Determinantes da procura de atendimento de urgência pelos usuários nas unidades de pronto atendimento da Secretaria Municipal de Saúde de Belo Horizonte [dissertação]. Belo Horizonte (MG): Universidade Federal de Minas Gerais, Programa de Pós-graduação em Enfermagem; 2005.

7. Rocha RLP. Percepções dos profissionais da atenção básica sobre o serviço de atendimento móvel de urgência de Belo Horizonte [dissertação]. Belo Horizonte (MG): Universidade Federal de Minas Gerais, Programa de Pós-graduação em enfermagem; 2009.

8. Nóbrega SM. Sobre a teoria das representações sociais. In: Moreira, ASP, Jesuíno, JC. Representações Sociais: teoria e prática. João Pessoa (PB): Universitária; 2003. p.57-87.

9. Moscovici S. A representação social da psicanálise. Rio de Janeiro (RJ): Zahar, 1978.

10. Abric JC. A abordagem estrutural das representações sociais. In: Moreira, ASP, Oliveira DC, organizadores. Estudos interdisciplinares de representação social. Goiânia (GO): AB Editora, 1998. p.271-283.

11. Ministério da Saúde (BR), Conselho Nacional de Saúde, Comissão Nacional de Ética em Pesquisa.
Resolução No 196 de 10 de outubro de 1996: diretrizes e normas regulamentadoras de pesquisa envolvendo seres humanos. Brasília (DF): MS; 1996.

12. Wachelke JFR, Camargo BV. Representações sociais, representações individuais e comportamento. Interam J. Psychol. 2007 Dez; 41(3):379-90.

13. Martins PPS. Atendimento pré-hospitalar: atribuição e responsabilidade de quem? Uma reflexão crítica a partir do serviço do corpo de bombeiros e das políticas de saúde "para" o Brasil à luz da filosofia da práxis [dissertação]. Florianópolis (SC): Universidade Federal de Santa Catarina. Programa de Pós-graduação; 2004.

14. Araujo MT, Alves M, Velloso ISC, Rocha TB, Gomes GG. O Serviço de Atendimento Móvel de Urgência de Belo Horizonte na percepção dos Enfermeiros. In: Anais do $61^{\circ}$ Congresso Brasileiro de Enfermagem, 2009 Dez 07-10; Fortaleza, Brasil. Fortaleza (CE): CBEN; 2009

15. Magalhães HM. Urgência e emergência: a participação do município. In: Reis, AT. Sistema Único de Saúde de Belo Horizonte: reescrevendo o público. São Paulo (SP): Xamã, 1998. p. 265-86.

16. Conselho Federal de Medicina. Resolução n. ${ }^{\circ}$ 1.451, de 10 de março de 1995. Define os conceitos de urgência e emergência e equipe médica e equipamentos para os pronto-socorros. Diário Oficial da União, Brasília, DF, 17 mar. 1995.

17. Jacquemot GA. Urgências e emergências em saúde: perspectivas de profissionais e usuários. Rio de Janeiro (RJ): Editora Fiocruz; 2005.

18. Pereira WAP, Lima MADS. O trabalho em equipe no atendimento pré-hospitalar à vítima de acidente de trânsito. Rev Esc Enferm. USP. 2009 Jun; 43(2):320-7.

19. Azevedo TMVE, Silva MJP. Relacionamento interpessoal no atendimento pré-hospitalar: uma revisão bibliográfica. Revista Nursing. 2008; 11(125):455-60.

20. Gentil RC, Ramos LH, Whitaker IY. Capacitação de enfermeiros em atendimento pré-hospitalar. Rev Latino-am Enfermagem. 2008 Abr; 16(2):192-7.

21. Sá MC, Carreteiro TC, Fernandes, MIA. Limites do cuidado: representações e processos inconscientes sobre a população na porta de entrada de um hospital de emergência. Cad Saúde Pública. 2008 Jun; 24(6):1334-43.

22. Garlet ER, Lima MAD, Santos JLG, Marques GQ. Organização do trabalho de uma equipe de saúde no atendimento ao usuário em situações de urgência e emergência. Texto Contexto Enfermagem. 2009 Abr-Jun; 18(2):266-72. 